\title{
COMPARAÇÃO DA INSEMINAÇÃO CONVENCIONAL E PÓS-CERVICAL SOBRE A EFICIÊNCIA REPRODUTIVA DE SUÍNOS
}

Andrea Francisca Araujo, Jeferson Corrêa Ribeiro, Andreia Santos Cezário, Crislaine Messias de Souza, Wallacy Barbacena Rosa Dos Santos, Aline Sousa Camargos

'Instituto Federal de Educação Ciência e Tecnologia Goiano - IF Goiano, Departamento de Zootecnia, Morrinhos, GO. E-mail: aline.camargos@ifgoiano.edu.br

\section{RESUMO}

Este trabalho objetivou comparar o efeito da inseminação artificial (IA) convencional e pós-cervical (PC) sobre a eficiência reprodutiva de suínos, avaliando taxa de prenhez, ocorrência de falhas reprodutivas e índices de leitões. O estudo foi conduzido em granja comercial, onde os dados de 364 matrizes suínas foram distribuídos aleatoriamente em três grupos: Convencional $(n=153)$ - IA convencional 12 e 24 horas após a detecção do cio; PC24 $(n=77)$ - IA pós-cervical 12 e 36 horas após a detecção de cio; e PC12 $(n=$ 134) - IA pós-cervical 12 e 24 horas após a detecção de cio. Foi registrada a ocorrência das falhas reprodutivas: aborto, micro-aborto e repetição de cio. As gestações e partos foram acompanhados e os leitões nascidos foram avaliados. Os dados foram registrados em software especializado (AGRINESS S2 versão 5.5.6). As taxas percentuais das falhas reprodutivas observadas e os dados foram submetidos à análise estatística pelo teste de Kruskal-Wallis (SAS, 2008), ao nível de significância de $5 \%$. As taxas de aborto e repetição de cio observadas foram baixas e não diferiram entre os grupos de tratamento $(p<0,05)$. A taxa de micro-aborto foi baixa no grupo PC12 e inexistente no grupo PC24, ambos inseminados pela técnica pós-cervical. No entanto, a taxa de micro-aborto do grupo CV foi maior que as demais $(p>0,05)$, mostrando maior taxa de micro-aborto em matrizes suínas submetidas a IA convencional. Não foram observados leitões mortos ao nascer durante o período do estudo. $O$ número de natimortos, mumificados e o peso médio dos leitões não diferiram significativamente entre os grupos avaliados $(p<0,05)$. 0 número de leitões nascidos vivos e o peso da leitegada foram significativamente maiores no grupo PC12 ( $p>0,05)$ comparado aos demais. Conclui-se que a IA pós-cervical com intervalo de 12 horas é a que mais contribuiu para a eficiência reprodutiva de matrizes suínas.

Palavras-chave: falhas reprodutivas; gestações; inseminação pós-cervical; leitões; matrizes.

\section{COMPARISON OF CONVENTIONAL AND POST CERVICAL INSEMINATION ON THE SWINE REPRODUCTIVE EFFICIENCY}

\section{ABSTRACT}

The objective of this study was to compare the effect of conventional and post cervical artificial insemination (Al) on the reproductive efficiency of swines, evaluating pregnancy rate, occurrence of reproductive failures and piglet indexes. The study was conducted in a commercial farm, where data from 364 matrices were randomly distributed into three groups: Conventional $(n=153)$ - conventional Al 12 and 24 hours after the detection of estrus; PC24 $(n=77)$ - post cervical IA 12 and 36 hours after oestrus detection; and PC12 ( $n=134)$ post-cervical IA 12 and 24 hours after oestrus detection. Occurrence of reproductive failures was recorded such as: abortion, micro-abortion and heat repetition. Gestations and deliveries were followed and the piglets born were evaluated. Data were recorded in specialized software (AGRINESS S2 version 5.5.6). Percentages of observed reproductive failures and data were submitted to statistical analysis by the Kruskal-Wallis test (SAS, 2008), at a significance level of $5 \%$. The observed abortion and estrus rates were low and did not differ between treatment groups $(p<0.05)$. Micro-abortion rate was low in the PC12 group and non-existent in the PC24 group, both inseminated by the post-cervical technique. However, the micro-abortion rate of the CV group was higher than the others ( $p>0.05$ ), showing a higher rate of micro-abortion in swine matings submitted to conventional Al. No dead piglets were observed at birth during the study period. Number of stillbirths, mummified and the average weight of the piglets did not differ significantly between the evaluated groups $(p<0.05)$. Number of live-born piglets and 
litter weight were significantly higher in the PC12 group $(p>0.05)$ compared to the others. It was concluded that post-cervical Al with a 12 hour interval is the one that most contributed to the reproductive efficiency of swine matrices.

Keywords: reproductive failures; gestations; post cervical insemination; piglets; matrices.

\section{INTRODUÇÃO}

A técnica da inseminação artificial (IA) em suínos data-se desde a década de 30 , porém foi desenvolvida e comercialmente aplicada na indústria apenas na década de 80 (ROCA et al., 2006). Utilizada em todo o mundo, com o intuito de obter maior eficiência reprodutiva e econômica nas granjas. Segundo Riesenbeck (2011), em torno de $60 \%$ do plantel mundial é inseminado artificialmente, destacando o Brasil e o Chile, que tiveram um aumento tanto na produção de suínos como no número de fêmeas atendidas pela técnica. Wentz et al. (2000) estimaram a realização de 1,6 milhões de primeiras IAs no Brasil, correspondendo a utilização da biotecnologia em praticamente $50 \%$ do plantel brasileiro das matrizes suínas em sistema tecnificado (MIELE; MACHADO, 2006). Trata-se de uma biotecnologia reprodutiva presente na produção suína, que maximiza o potencial genético do reprodutor suíno, e reduz a introdução de doenças nas granjas (ANTUNES, 2004), sendo considerada de grande importância para a suinocultura (WENTZ et al., 2000; MIELE; MACHADO, 2006).

Com retorno das pesquisas sobre a IA pela comunidade científica da suinocultura no início de 2000, realizaram-se uma série de trabalhos já em condições de campo e com equipamentos utilizados atualmente (DALLANORA, 2013). Permitindo o aumento da utilização de inseminação pós-cervical, que tratase da introdução do sêmen na fêmea suína ultrapassando as ondulações da cérvix, realizada com catéteres capazes de depositar o sêmen no corpo do útero (WATSON et al., 2001; WATSON; BEHAN, 2002; SERRET et al., 2005). A inseminação pós-cervical é uma técnica com metodologia simples, pode ser aplicada com o treinamento dos funcionários da própria granja sem muitas dificuldades (WATSON; BEHAN, 2002; VAZQUEZ et al., 2008).

O mercado suinícola está crescendo e a implementação de técnicas para melhor eficiência reprodutiva nos suínos está permitindo determinar melhores índices, tais como o número de leitões desmamados/fêmea/ano, que sofre influência de diversos aspectos, desde o momento de realização da $\mathrm{I} A /$ cobertura. Observando ainda, o período da duração do estro e o momento da ovulação que desempenham papel fundamental (BORTOLOZZO, 1993) para resultados satisfatórios do método.

Neste contexto, este trabalho teve por objetivo comparar o efeito da IA convencional e pós-cervical sobre a eficiência reprodutiva de suínos, avaliando taxa de prenhez, ocorrência de falhas reprodutivas e índices de leitões.

\section{MATERIAL E MÉTODOS}

O presente experimento foi conduzido na granja comercial do Sítio da Gameleira, situado no município de Lagoa Dourada - MG, mesorregião do Campo das Vertentes no decorrer do ano de 2014. O município apresenta latitude S 20 54' 52" e longitude W 44 04' 42", em região montanhosa (70\%) com clima tropical de altitude, caracterizado por verões quentes e úmidos e média térmica anual de 19,3ํ․

O banco de dados utilizado continha 364 fêmeas em idade reprodutiva, com ciclos estrais regulares e sem histórico de doenças. Para a coleta do sêmen, foram utilizados três reprodutores, alternadamente e o ejaculado avaliado no Laboratório de Sêmen.

Para a inseminação, foram usados somente os ejaculados refrigerados com aspecto normal e motilidade maior que $70 \%$. As doses tiveram sua concentração espermática de 8 bilhões para inseminação convencional e padronizada para a inseminação pós-cervical em 4 bilhões. Para tanto, foi adicionado o diluente industrializado (MRA média conservação, Consuitec). A identificação do cio das matrizes se fez pelo uso do rufião, passado no corredor entre as baias onde estavam as mesmas e identificando o cio ao parar em uma das baias das matrizes. Para o diagnóstico de gestação, repetia-se o procedimento inferindo que a fêmea não estaria prenha, se o macho parasse na baia.

A inseminação das matrizes se deu por duas formas: convencional e pós-cervical. Para a 
inseminação convencional, foi utilizado o método de deposição do sêmen no interior da cérvix com pipeta de IA para suínos (pipeta descartável ponta espiral, Consuitec). Já para a IA póscervical, a deposição de sêmen foi intrauterina utilizando-se sistema de passagem de cateter (pipeta descartável ponta de espuma para porca, Consuitec).

O banco de dados foi distribuído em três grupos de tratamento, de acordo com o tipo e intervalo entre IAs: CV ( $n=153)$ - IA convencional 12 e 24 horas após a detecção do cio; PC24 ( $\mathrm{N}=77)$ - IA pós-cervical 12 e 36 horas após a detecção de cio; e PC12 ( $n=134)$ - IA pós-cervical após 12 e 24 horas após a detecção de cio.

As matrizes inseminadas foram submetidas a diagnóstico de gestação e acompanhadas durante todo o período gestacional. Toda e qualquer anormalidade e ocorrência de falhas reprodutivas (aborto, microaborto e repetição de cio) foi registrada por técnico da granja. Após o nascimento, os leitões foram contados, pesados e avaliados. Observado e registrado: o número de leitões nascidos vivos, o peso da leitegada, o peso médio dos leitões, o número de mortos ao nascer, ocorrência de natimortos e de mumificados.

Os dados reprodutivos e dos leitões foram registrados em software especializado (AGRINESS S2 versão 5.5.6) por técnico da granja. Para as análises estatísticas, como os dados não seguiram uma distribuição normal, as taxas percentuais das falhas reprodutivas observadas (aborto, micro-aborto e repetição de cio) e as médias de cada grupo (taxa de prenhez, número de leitões nascidos vivos, peso da leitegada, peso médio dos leitões, número de mortos ao nascer, natimortos e mumificados) foram submetidas ao teste de Kruskal-Wallis (SAS, 2008), ao nível de significância de 5\%.

\section{RESULTADOS E DISCUSSÃO}

IA convencional $x$ pós-cervical

A IA convencional apresentou a menor taxa de gestação $(p>0,05)$ tabela 1 , porque após a inseminação pode ocorrer fagocitose do ejaculado ou o refluxo da dose inseminada, diminuindo assim a taxa de prenhez. Dentre os grupos de IA pós-cervical, o grupo PC12 obteve maior taxa de prenhez $(p<0,05)$, pois a perca do ejaculado é menor em relação a IA convencional.

Tabela 1. Taxa de prenhez (em porcentagem) em matrizes suínas após IA convencional e pós-cervical (com intervalos de 12 e 24 horas).

\begin{tabular}{lcc}
\hline Tratamento & $\mathrm{N}$ & Taxa de Gestação* \\
\hline CV & 153 & $89,95 \mathrm{c}$ \\
PC24 & 77 & $95,60 \mathrm{~b}$ \\
PC12 & 134 & $97,40 \mathrm{a}$ \\
\hline
\end{tabular}

*Letras diferentes na mesma coluna diferem significativamente entre si $(p<0,05)$. $N=$ número de matrizes; $C V=$ inseminação artificial convencional; PC24 = inseminação artificial pós-cervical com 24 horas de intervalo; e PC12 = inseminação artificial pós-cervical com 12 horas de intervalo.

Assim, após as análises, pode-se dizer que a inseminação é a base para se melhorar a eficiência reprodutiva nos suínos. Os principais efeitos da inseminação pós-cervical dependem de diversos fatores, como a quantidade de espermatozoides presentes no sêmen. Estes fatores são determinantes para que haja uma prenhez satisfatória. Quando o sêmen é depositado no trato reprodutivo da fêmea suína, são desencadeados mecanismos de defesa do organismo da fêmea. O sêmen é reconhecido como um material estranho depositado no útero. Ocorrendo uma diminuição do volume e do número de espermatozóides (MATTHIJS et al., 2003).

Como já dito, a IA pós-cervical possui variadas vantagens que reduzem perdas econômicas e materiais, uma delas é a redução das perdas de espermatozóides por refluxo e fagocitose, devido ao trajeto percorrido pelas células espermáticas até o oviduto que seria menor e à cérvix, principal barreira mecânica para o trânsito espermático, que já teria sido ultrapassada (BORTOLOZZO et al., 2003). Portanto, nesta técnica poderiam ser utilizadas doses inseminantes com reduzida concentração de espermatozóides e com menor volume total, tendo aproveitamento mais eficiente dos ejaculados e menor desgaste dos machos.

Diferente dos resultados deste estudo, Bolarin (2006) afirma que, ao comparar dados reprodutivos das inseminações convencional e pós-cervical, pode-se verificar que alguns aspectos não são significativamente diferentes. 
Inferindo que a inseminação pós-cervical pode não ter efeito sobre o potencial de fertilidade das fêmeas. Outros fatores podem afetar a prenhez das fêmeas por inseminação convencional, tais como a escolha da matriz, a viabilidade espermática e o ambiente.

\section{Falhas reprodutivas}

As taxas de aborto e repetição de cio observadas neste estudo foram baixas, de acordo com o método estatístico utilizado (tabela 2) e não diferiram significativamente entre os grupos de tratamento $(p<0,05)$.
Portanto, segundo Mellagi et al. (2006), os abortamentos contribuem negativamente para os índices reprodutivos da suinocultura. Pois com a perda da prenhez, há diminuição da taxa de parto, com consequente queda no número de leitões produzidos ao ano. Além disso, contribuem para o aumento dos dias não produtivos (DNP), representando perdas econômicas e aumentando os custos de produção.

Tabela 2. Ocorrência de falhas reprodutivas em matrizes suínas após IA convencional e pós-cervical (com intervalos de 12 e 24 horas).

\begin{tabular}{lccc}
\hline Tratamento & Aborto & Repetição de cio & Micro-aborto* \\
\hline CV & $0,79 \%$ & $0,79 \%$ & $8,47 \% \mathrm{a}$ \\
PC24 & $0 \%$ & $4,4 \%$ & $0 \% \mathrm{~b}$ \\
PC12 & $1,30 \%$ & $0 \%$ & $1,30 \% \mathrm{~b}$ \\
\hline
\end{tabular}

*Letras diferentes na mesma coluna diferem significativamente entre si $(p<0,05)$. $N=$ número de matrizes; $C V=$ inseminação artificial convencional; PC24 = inseminação artificial pós-cervical com 24 horas de intervalo; e PC12 = inseminação artificial pós-cervical com 12 horas de intervalo.

Contudo, a repetição de cio apesar de não apresentar diferença estatística nos dados utilizados deve ser mantida atenção sobre a matriz inseminada para que não haja percas diretas e indiretas com o plantel, ou seja, a matriz inseminada é mantida no plantel e traz custos ao produtor, com alimentação, tempo, ambiente e com a dose de sêmen que será desperdiçada ao realizar a aplicação da técnica de IA na matriz, devendo ser descartada posteriormente se houver mais ocorrência com a mesma matriz.

Ainda segundo a fisiologia da matriz, Sobestiansky et al. (1998) realizaram pesquisas e mostraram que a ovulação influencia a repetição de cio, por ocorrer aproximadamente aos $70 \%$ do período do cio, que pode ser de 48 a 56 horas, variando de acordo com a fisiologia da porca. Sendo que a fêmea suína é poliéstrica não sazonal com múltiplas ovulações e ciclos estrais médios de 21 dias, podendo variar de 18 a 23 dias. Apresentando cio com duração de 12 a 72 horas, tendo maiores durações em porcas mais velhas. Destacando assim a importância de observação atenta do granjeiro para que a fêmea seja identificada e retirada do plantel.

Wentz et al. (2008) e Sobestiansky et al. (2012) classificam a repetição de cio após a inseminação artificial em: 1) retornos cíclicos ou regulares, acontecendo 21 dias após a mesma; 2 ) retornos irregulares ou acíclicos, com intervalos superiores a 24 dias após a inseminação.

Ainda, os retornos regulares mostram a ocorrência de falha na fecundação, morte de todos os conceptos ou a permanência de menos de cinco embriões ao primeiro sinal de gestação, detectados 12 dias após a fecundação. A taxa aceitável para que ocorra em um plantel é de 4 a $8 \%$, por Wentz et al. (2008). Entretanto, para a ocorrência dos retornos irregulares, aceita-se taxa de 2 a 4\%, segundo Meredith (1995), por serem relacionados a falhas na manutenção da gestação.

Para o índice em granjas tecnificadas, o total de retorno ao cio após a cobertura ou inseminação artificial varia de 6 a 8\% (WENTZ et al., 2008) até 5 a 15\% (SOBESTIANSKY et al. (2012).

Já a falha reprodutiva micro-aborto ocorre no início da gestação de forma discreta, inferindo que o funcionário responsável deve estar atento a todo instante nas primeiras semanas após o diagnóstico de gestação. Foi observada neste estudo diferença significativa $(p<0,05)$ entre os grupos de tratamento para esta falha. A taxa de micro-aborto foi baixa no grupo PC12 e inexistente no grupo PC24, ambos inseminados pela técnica pós-cervical. No entanto, foi significativamente maior no grupo CV 
$(p>0,05)$, indicando que a técnica pós-cervical permite controlar a ocorrência desta falha.

O uso da IA pós-cervical neste estudo não aumentou a ocorrência de falhas reprodutivas. Demonstrou que a técnica pode ser incluída na granja sem que haja perdas significativas em relação a IA convencional.

Contudo, outros autores, como Watson e Behan (2002), Dallanora et al. (2004), Bennemann (2004), Mezalira (2005), relataram não observar diferenças no desempenho e na ocorrência de falhas reprodutivas entre fêmeas inseminadas pelas técnicas de IA pós-cervical e a IA convencional. Estes afirmam que não há prejuízo nos índices produtivos com o emprego da inseminação intrauterina, podendo até apresentar mais vantagens em sua utilização.

Efeito sobre os leitões e suas características

Não foram observados leitões mortos ao nascer durante o período do estudo (Tabela 3). 0 número de natimortos, mumificados e o peso médio dos leitões não diferiram significativamente $(p<0,05)$ entre os grupos de tratamento. $\mathrm{O}$ número de leitões natimortos e mumificados presentes observados no banco de dados foi baixo, levando a não diferença estatística.

Tabela 3. Efeito das inseminações convencional (CV) e pós-cervical com intervalo de 24 horas (PC 24) e 12 horas (PC 12) sobre o nascimento de leitões e suas características.

\begin{tabular}{lccccc}
\hline Tratamento & Natimortos & Mumificados & $\begin{array}{c}\text { Peso médio } \\
\text { dos leitões }\end{array}$ & $\begin{array}{c}\text { Número nascidos } \\
\text { vivos }\end{array}$ & $\begin{array}{c}\text { Peso da } \\
\text { leitegada }\end{array}$ \\
\hline CV & 0,84 & 0,39 & 1,37 & $13,04 \mathrm{~b}$ & $17,63 \mathrm{~b}$ \\
PC24 & 0,85 & 0,41 & 1,36 & $13,65 \mathrm{~b}$ & $18,36 \mathrm{~b}$ \\
PC12 & 1,06 & 0,29 & 1,35 & $14,44 \mathrm{a}$ & $19,44 \mathrm{a}$ \\
\hline
\end{tabular}

*Letras diferentes na mesma coluna diferem significativamente entre si $(\mathrm{p}<0,05)$. $\mathrm{N}=$ números de Matriz; $\mathrm{CV}=$ Inseminação Artificial Convencional; PC24 = Inseminação Artificial pós-cervical 24 horas de intervalo; PC12 = Inseminação Artificial pós-cervical 12 horas de intervalo.

A natimortalidade está associada a fatores relacionados ao evento do parto, como: ambiente, nutrição e toxicoses; ou fatores ligados ao macho ou à fêmea que produziram a leitegada (CHRISTIANSON, 1992). A taxa de natimortos observada pode ser reflexo de um bom manejo e alimentação adequada.

De acordo com os estudos de Borges et al. (2003), a mumificação ocorre em 50\% das leitegadas com mais de 12 leitões, como neste estudo. Assim, Zanella et al. (2007) relatam a mumificação como um processo não específico, pois acontece com a morte dos fetos e sua retenção dentro do útero, desidratando-se. Ainda, relataram que a fase de ossificação do esqueleto ocorre por volta dos 35 dias de gestação. Com a morte do feto neste período, há a ocorrência do processo de mumificação.

O número de leitões nascidos vivos e o peso da leitegada foram significativamente maiores no grupo PC12 $(p>0,05)$ comparado aos demais. O peso da leitegada foi maior neste grupo devido ao maior número de leitões vivos. 0 depósito de sêmen intrauterino, pela técnica póscervical, pode ter favorecido a fertilização quando aliado ao intervalo de 12 horas entre as duas IAs, o que aperfeiçoou o encontro de espermatozoides viáveis com os oócitos.

Números semelhantes foram obtidos em um estudo com suínos cruzados F1 (Large White $x$ Landrace) e Large White por Corrêa et al. (2003), onde foi observada uma média de peso de 14,10 kg e tamanho da leitegada ao nascer de 10,8 leitões.

Hafez (2004) relata que, em relação ao desempenho reprodutivo de suínos, o número de leitões vivos no parto se torna relevante para se obter uma medida para tal característica, onde pode ser considerado ainda o número de leitões à desmama e o peso total dos mesmos, sendo estes produzidos por porca/ano.

Contudo, ao ser utilizada a IA póscervical com intervalo de 12 horas, pode-se relevar o aspecto da fertilização em favor do tipo de técnica a ser utilizada. Pois, ao realizar o depósito de sêmen dentro do útero, podemos ver que demonstrou redução em taxas de falhas reprodutivas como as taxas de aborto, repetição de cio e micro-aborto. Revelando assim, que sua utilização torna-se eficaz como prevenção de falhas reprodutivas que as granjas e pesquisadores tanto estudam para que diminua a incidência. 
Portanto, a IA convencional, mesmo sendo a mais utilizada por muitas granjas, apresentou uma maior taxa de micro-aborto, o que gera prejuízo à granja, levando os produtores a repensar a sua utilização. Assim, a IA póscervical relacionada a quantidade de vezes e horas, mostrou-se eficiente quando realizada duas vezes e com intervalo de 12 horas. Foi o melhor protocolo dentro deste método de inseminação, incrementando a eficiência reprodutiva das matrizes suínas.

\section{CONCLUSÃO}

A técnica de inseminação pós-cervical apresentou melhores resultados, pois esta obteve maior taxa de prenhez. Com isso, gerou maior número de leitões nascidos vivos, elevando seus índices em relação ao da IA convencional.

\section{AGRADECIMENTOS}

Ao Sítio da Gameleira, que viabilizou a execução do estudo.

\section{REFERÊNCIAS}

ANTUNES, R. C. Avanços e perspectivas do melhoramento genético de suínos. 2004.

BENNEMANN, P. E. Reproductive performance of the sows undergoing intrauterine insemination in the preovulatory different intervals. Animal Reproduction, v.1, n.1, p.106-110, 2004.

BOLARIN, A.; ROCA, J.; RODRIGUEZ-MARTINEZ, H.; HERNANDEZ, M.; VAZQUEZ, J. M.; MARTINEZ, E. A. Dissimilarities in sows, ovarian status at the insemination time could explain differences in fertility between farms when frozen-thawed semen is used. Theriogenology, v.65, p.669-680, 2006.

https://doi.org/10.1016/j.theriogenology.2005.0 $\underline{6.006}$

BORGES, V. F.; WEBER, D.; SOUZA, L. P. Importância da mumificação fetal na suinocultura moderna. In: CONGRESSO BRASILEIRO DE VETERINARIOS ESPECIALISTAS EM SUINOS, 11. Anais... 2003. p.195-196.

BORTOLOZZO, F. P. Implicações da duração do estro e momento da ovulação na eficiência reprodutiva de suínos. In: CONGRESSO BRASILEIRO DE VETERINÁRIOS ESPECIALISTAS EM SUÍNOS, 6. Anais... Associação Brasileira de
Veterinários Especialistas em Suínos, 1993. p. 6474.

BORTOLOZZO, F. P.; DALLANORA, D.; BERNARDI, M. L.; BENNEMANN, P. C.; BORTOLOZZO, F. P. Técnicas associadas à inseminação artificial no suíno que visam à redução do número de espermatozoides necessários por fêmea ao ano. Revista Brasileira de Reprodução Animal, v.27, p.133-139, 2003.

CHRISTIANSON, W. T. Stillbirths, mummies, abortions and early embryonic death. In: TUBBS, R. C.; LEMAN, A. D. Veterinary Clinics of North America: food animal practice. Swine Reproduction, Philadelphia, v.8, n.3, p.623-639. $1992 . \quad$ https://doi.org/10.1016/S07490720(15)30708-8

CORRÊA, M. N.; LUCIA, J. T.; BIANCH, I.; DONIN, M. A.; ARRIADA, A. A.; DESCHAMPS, J. C.; MEINCKE, W. Natimortalidade em suínos. Distribuição de potenciais fatores de risco. In: REUNIÃO ANUAL DA SOCIEDADE BRASILEIRA DE ZOOTECNIA, 40. Anais... Santa Maria: Sociedade Brasileira de Zootecnia, 2003. CD-ROM.

DALLANORA, D.; MEZALIRA, A.; KATZER, L. H. Desempenho reprodutivo de fêmeas suínas inseminadas pela técnica intra-uterina ou tradicional. Pesquisa Agropecuária Brasileira, v.39, n.8, p.815-819, 2004. https://doi.org/10.1590/S0100$\underline{204 X 2004000800013}$

DALLANORA, D. Técnica pós-cervical muda padrões de inseminação artificial. 0 Presente Rural, 2013.

HAFEZ, E. S. E.; HAFEZ B. Reprodução animal. 7. ed. São Paulo: Manole, 2004.

MATTHIJS, E.; WOELDERS, H.; ENGEL, B. Neutrophil recruitment and phagocytosis of boar spermatozoa after artificial insemination of sows, and the effects of inseminate volume, sperm dose and specific additives in the extender. Jornal of Reproduction and Fertility, v.125, p.357-367, 2003. https://doi.org/10.1530/rep.0.1250357

MELLAGI, A. P. G.; BORTOLOZZO, F. P.; WENTZ, I.; Abortamento e partos antecipados em suínos. I Simpósio UFRGS sobre produção, reprodução e sanidade suína. 2006. p. 215-220. 
MEREDITH, M. J. Pig Reproduction and Infertility. In: MEREDITH, M.J. (Eds). Animal breeding and infertility. Cambridge: Blackwell Science, p.278353, 1995.

MEZALIRA, A. Influence of dose sperm cells and post- insemination reflux on reproductive performance intrauterine insemination nuts. Domestic Animal Reproduction, v.40, p.1-5, $2005 . \quad$ https://doi.org/10.1111/j.1439$\underline{0531.2004 .00524 . x}$

MIELE M.; MACHADO, J. S. (Eds.). Levantamento sistemático da produção e abate de suínos. Concórdia: Embrapa Suínos e Aves, 2006. https://doi.org/10.1111/i.1439-

0531.2004.00524.x

RIESENBECK, A. Review on international trade with boar sêmen. Reproduction in Domestic Animals, v.46, n.(Suppl 2), p.1-3, 2001. https://doi.org/10.1111/j.1439-

0531.2011.01869.x

ROCA, J.; VAZQUÉZ, J. M.; GIL, M. A.; CUELLO, C.; PARRILLA, I.; MARTíNEZ, E. A. Challenges in artificial pig insemination. Reproduction in Domestic Animals, v.2, p.43-53, 2006. https://doi.org/10.1111/j.1439-

0531.2006.00768.x

SAS. Método Kruskal-Wallis. Programa Statistical Analysis System, 2008.

SERDIUK, S. I. Artificial insemination of pigs. Moscow: Kolos, 1970. 144p.

SERRET, C. G. Intrauterine artificial insemination in female swine with distinct sperm concentrations, parities and methods of ovulation estimation. Animal Reproduction, v.2, p.250-256, 2005.

SOBESTIANSKY, J.; BARCELLOS, D. Doenças dos Suínos. 2. ed. Goiana: Cânone, 2012. 959p.

SOBESTIANSKY, J.; WENTS, I.; SILVEIRA, P. R. S. Suinocultura intensiva: produção, manejo e saúde do rebanho. Brasília: Embrapa, 1998. $388 p$.

VAZQUEZ, J. M.; ROCA, J.; GIL, M. A. Low-Dose Insemination in Pigs: Problems and Possibilities. Reproduction in Domestic Animals, v.43, n.2, p.347-354, 2008. https://doi.org/10.1111/j.14390531.2008.01183.x

ZANELLA, E.; SILVEIRA, P. R. S.; SOBESTIANSKY. J. 2007. Natimorto, Mumificados e Natimortalidade. In: SOBESTIANSKY, J.; BARCELLOS D.E.S.N. (Eds). Doenças de suínos. Goiânia: Cânone, 2007. p.564-567.

WATSON, P. F.; BEHAN, J. R. Intrauterine insemination of sows with reduced sperm numbers: results of a commercially-based field trial. Theriogenology, v.57, p.1683-1693, 2002. https://doi.org/10.1016/S0093-691X(02)00648-9

WATSON, P. F.; BEHAN, J. R.; DECUADROHANSEN, G.; CASSOU, B. Deep insemination of sows with reduced sperm numbers does not compromisse fertility: a commercially-based field trial. In: INTERNATIONAL CONFERENCE ON PIG REPRODUCTION, 6. Proceedings... University of Missouri-Columbia, 2001. p. 135.

WENTZ, I.; HEIM, G.; BORTOLOZZO, F. P. Como avaliar e interpretar o aumento dos retornos ao estro após a inseminação artificial na suinocultura. Acta Scientiae Veterinariae, Porto alegre, v.36, 2008. Disponível em: $<$ http://www.ufrgs.br/actavet/36-suple1/09_retornos.pdf>. Acesso em: 01 dez. 2016.

WENTZ I.; VARGAS A. J.; BORTOLOZZO F.; CASTAGNA C. D. Situação atual da inseminação artificial em suínos no Brasil e viabilização econômica dessa biotécnica. In: SIMPÓSIO INTERNACIONAL MINITUB INSEMINAÇÃO ARTIFICIAL EM SUÍNOS, 3. Anais... Flores da Cunha, 2000. p.5-12.

Recebido para publicação em 16/08/2017

Revisado em 13/09/2017

Aceito em 09/10/2017 